\title{
Die österreichische Pestkommission in Bombay 1897 und die letzten Pest-Todesfälle in Wien 1898
}

\author{
Heinz Flamm
}

Eingegangen: 15. März 2018 / Angenommen: 2. August 2018 / Online publiziert: 29. August 2018

(C) Der/die Autor(en) 2018

\begin{abstract}
Zusammenfassung Eine ärztliche Kommission der Österreichischen Akademie der Wissenschaften untersuchte Kranke während der Pest-Epidemie in Bombay (Mumbai) im Jahre 1897. Die klinischen, pathologischanatomischen, histologischen und bakteriologischen Befunde wurden danach publiziert. Mit den nach Wien gebrachten Pestbakterienstämmen wurden bis im Oktober 1898 bakteriologische Untersuchungen und Versuche mit verschiedenen Tierarten durchgeführt. Am Ende dieser Forschungsarbeiten infizierte sich der Laboratoriumsdiener Franz Barisch unbemerkt. Seine Behandlung wurde vom Delegierten der Pestkommission, Dozent Dr. Hermann Franz Müller, und zwei Pflegerinnen übernommen. Barisch starb vier Tage später, am 18. Oktober 1898, an einer schweren pulmonalen Infektion. Eine der Pflegerinnen, Albine Pecha, erkrankte zwei Tage danach. Sie und Dr. Müller wurden sofort in eine Isolierbaracke gebracht, wo Müller am selben Tag an Pest-Pneumonie erkrankte und nach drei Tagen, am 23. Oktober, starb. Die Pflegerin Albine Pecha hatte eine wahrscheinlich durch Pestseruminjektionen verzögerte und wechselnd schwer verlaufende Lungenpest. Sie starb zehn Tage nach Krankheitsbeginn, am 30. Oktober 1898.
\end{abstract}

Schlüsselwörter Pest · Pestkommission · Wien 1898 . Laboratoriumsinfektion

The Austrian Plague Commission in Bombay 1897 and the last fatal Plague Cases in Vienna 1898

Summary During the plague epidemic 1897 in Bombay a medical commission of the Austrian Academy of

Univ.-Prof. Dr. med. Dr.med. h.c. H. Flamm ( $ه)$ Martinstraße 7, 3400 Klosterneuburg, Österreich office.martinstr@aon.at
Sciences carried out clinical, pathologic-anatomical, -histological and bacteriological investigations. With the plague bacteria strains brought to Vienna bacteriological investigations and infection experiments with various species of animals were done. Finishing the animal investigations, the laboratory helper Franz Barisch caught a plague infection unnoticedly and four days later, on October 18th, 1898, he died with a heavy pulmonary affection. His treatment has been carried out by a delegate of the commission, Dozent Dr. Hermann Franz Müller, and two nurses. On October 20th one of them, Albine Pecha, fell ill. She and Dr. Müller immediately were isolated. On the same day, Dr. Müller showed symptoms of plague pneumonia. He died three days later, on October 23rd. Pecha's pulmonic plague went varying heavy and was prolonged probably by injections of plague serum. She died on October 30th, 1898.

Keywords Plague · Plague Commission • Vienna 1898 . Laboratory infection

Nach dem langsamen Rückzug der Reste der mittelalterlichen Pest ab etwa 1750 auf ihre alten enzootischen Dauerherde in Innerasien und Zentralafrika traten in der zweiten Hälfte des 19. Jahrhunderts vorerst nur mehr vereinzelte Ausbrüche menschlicher Pest an deren Rändern auf. Im Winter 1878/1879 suchte eine verheerende Pestepidemie im Gouvernement Astrachan die Orte beidseits der Wolga vor deren Mündung in das Kaspischen Meer heim. Einer von der deutschen Regierung veranlassten internationalen Kommission gehörten als österreichisch-ungarische Delegierte der Sanitätsreferent für Galizien Prof. Dr. Alfred Biesiadecki (1839-1889) und Dr. Kiemann an [1]. Als die Vereinigte Kommission, durch administrative Hindernisse verzögert, erst anfangs Februar 1879 das Seu- 
chengebiet erreichte, war die Pest bereits erloschen. Es konnten daher nur epidemiologische Fragen erörtert werden.

Österreichische Aktivitäten wurden wieder entfacht, nachdem sich in den 1890er-Jahren eine schwere Pest-Pandemie auszubreiten begonnen hatte. Diese entstand durch Ausbrüche aus dem asiatischen Hochland nach Osten und Süden. Durch Soldatenkarawanen verbreitet, erreichte die Pest 1894 Hongkong. Im selben Jahr wütete die Pest im Nordosten Indiens, von wo sie durch Pilger nach Süden verschleppt wurde und 1896 Bombay [Mumbai] erreichte. Insbesondere von den beiden Hafenstädten wurde die Seuche durch den starken Schiffsverkehr mit Personen und Waren in alle Erdteile verbreitet [2, 3]. In Europa konnten größere Ausbrüche durch genaue Kontrollen im Seeverkehr und durch Rattenbekämpfung in den Häfen zu meist verhindert werden. Die Pest-Pandemie ebbte in der ersten Hälfte des 20. Jahrhunderts aus. Enzootische Pestherde bestehen aber weiterhin in Asien und Afrika, sodass Einzelfälle und kleinere Epidemien auch noch im 21. Jahrhundert auftreten.

Die Pest-Pandemie 1896/97 veranlasste die österreichisch-ungarische Regierung, bei den Regierungen Europas anzuregen, dass die bereits 1892 in der 7. Internationalen Sanitätskonferenz zu Venedig beschlossenen Vorkehrungen gegen die Cholera sinngemäß auch für die Pest gelten sollen. Es wären also gemeinsame Vereinbarungen zu treffen, um einerseits wirksame, andererseits aber Handel und Verkehr wenig schädigende Maßnahmen gegen die Einschleppung der Seuche nach Europa sicherzustellen. Der Initiative Österreich-Ungarns entsprechend trat am 16. Februar 1897 die 10. Internationale Sanitätskonferenz in Venedig zusammen und beschloss den Antrag [4].

In der Stadt Bombay hatte die Epidemie in Winter 1896/97 10.606 Todesfälle verursacht [5]. Da die Erkrankung durch Pilger nach Mekka übertragen worden ist, entstand auch für Österreich-Ungarn die Gefahr einer Einschleppung der Infektion durch die in die Provinz Bosnien und Herzegowina heimkehrenden Mekka-Pilger. Der rege Schiffsverkehr aus Asien stellte eine weitere Bedrohung dar. Es stimmte deswegen der k.k. Oberste Sanitätsrat [OSR] in seiner Sitzung am 17. Oktober 1896 [6] den vom k.k. Ministerium des Innern [als oberste Gesundheitsbehörde] im Einvernehmen mit dem k.k. Handelsministerium erlassenen Maßnahmen zur „Hintanhaltung der Verschleppung der Pest“ durch den Schiffsverkehr zwischen Triest und Vorderindien zu. Die genaue Durchführung ist im Erlass der k. k. Seebehörde in Triest vom 20. Oktober 1896 festgelegt [7]. Tatsächlich erlag in Triest am 4. November 1896 ein kranker Bootsmann eines türkischen Dampfers der bakteriologisch verifizierten Pest, jedoch ohne weitere Infektionen verursacht zu haben [8]. Für das Hinterland galt der Erlass der k. k. n.-ö. Statthalterei vom 22. Jänner 1897 betreffend die Assanierung in den Gemeinden anlässlich der Pestgefahr [9]. Genauere Anweisungen für die Über- wachung der Einfuhr von Waren und Verkehrsgegenständen aus Südasien und der Ankunft und Weiterreise von Personen aus verseuchten asiatischen Gebieten folgten mit Erlass des k. k. Ministeriums des Inneren vom 19. März 1897 [10].

Die Epidemie erreichte mit ansteigenden Werten in den ersten Wochen des Jahres 1897 ihren Höhepunkt mit einer Letalität von 75-85\% [11]. Es befasste sich deswegen der OSR am 9. und 23. Jänner 1897 [12] weiterhin mit der Pestabwehr. Er bestätigte, dass die in den internationalen Sanitätskonferenzen zu Venedig (1892) und Dresden (1893) festgelegten Vorkehrungen gegen die Cholera auch gegen die Pest anzuwenden seien. Der OSR beschloss weiters seine Bereitschaft zur Unterstützung der Kaiserlichen Akademie der Wissenschaften, nachdem Prof. Dr. Anton Weichselbaum (1845-1920) mitgeteilt hatte, dass die Akademie demnächst drei Ärzte nach Bombay schicken wird.

\section{Die Pest-Kommission der Österreichischen Aka- demie der Wissenschaften}

Die sich im Jänner 1897 in den Wiener Tageszeitungen häufenden Berichte über die Pest in Indien waren der Anstoß für die Mathematisch-naturwissenschaftliche Klasse der Akademie der Wissenschaften, am 17. Jänner die Sendung einer Kommission nach Indien zur Erforschung der Krankheit zu beschließen. Gleichzeitig lud sie die Gesellschaft der Ärzte in Wien ein, allfällige Wünsche für besondere Untersuchungen mitzuteilen. Deren Verwaltungsrat nahm in seiner Sitzung am 29. Jänner das von ihm angeregte Referat des praktischen Arztes Dr. Simon Steinach (1834-1904) an, das eine mögliche Pesteinschleppung durch den Import von indischer Baumwolle behandelte [13].

Die ausgesuchten und am 24. Jänner zur Teilnahme eingeladen Herren, Privatdozent Dr. Hermann Franz Müller (1866-1898) von der I. Medizinischen Klinik (Prof. Dr. Herrmann Nothnagel, 1841-1905) sowie Dr. Heinrich Albrecht (1866-1922) und Dr. Anton Ghon (1866-1936), Assistenten des Pathologisch-anatomischen Instituts (Prof. Dr. Anton Weichselbaum, 1845-1920), erklärten sich dazu bereit. Ergänzt wurde die Expedition noch durch den Aspiranten Dr. Rudolf Pöch (1870-1921) von der II. Medizinischen Klinik (Prof. Dr. Edmund Neusser, 1852-1912), der auch ausgebildeter Fotograf war, und durch den geschulten Prosekturdiener des Wiener k.k. Kaiserin-ElisabethSpitals, Mathias Stöbich ([14], Abb. 1, 2 und 3).

Die Pestkommission hatte den Auftrag, ,in klinischer, anatomischer und bakteriologischer Hinsicht die in Bombay damals wüthende Volksseuche auf das genaueste zu studiren und zwar ganz im Geiste und nach der Methode, in welcher die genannten Disciplinen an der Wiener Schule tradirt werden“. Auch in epidemiologischer Beziehung sollten nach Möglichkeit Erfahrungen gesammelt werden, die in der Zukunft $\mathrm{zu}$ verwerten wären. Von den bakteriologischen Untersuchungen erwartete man sich weitere 


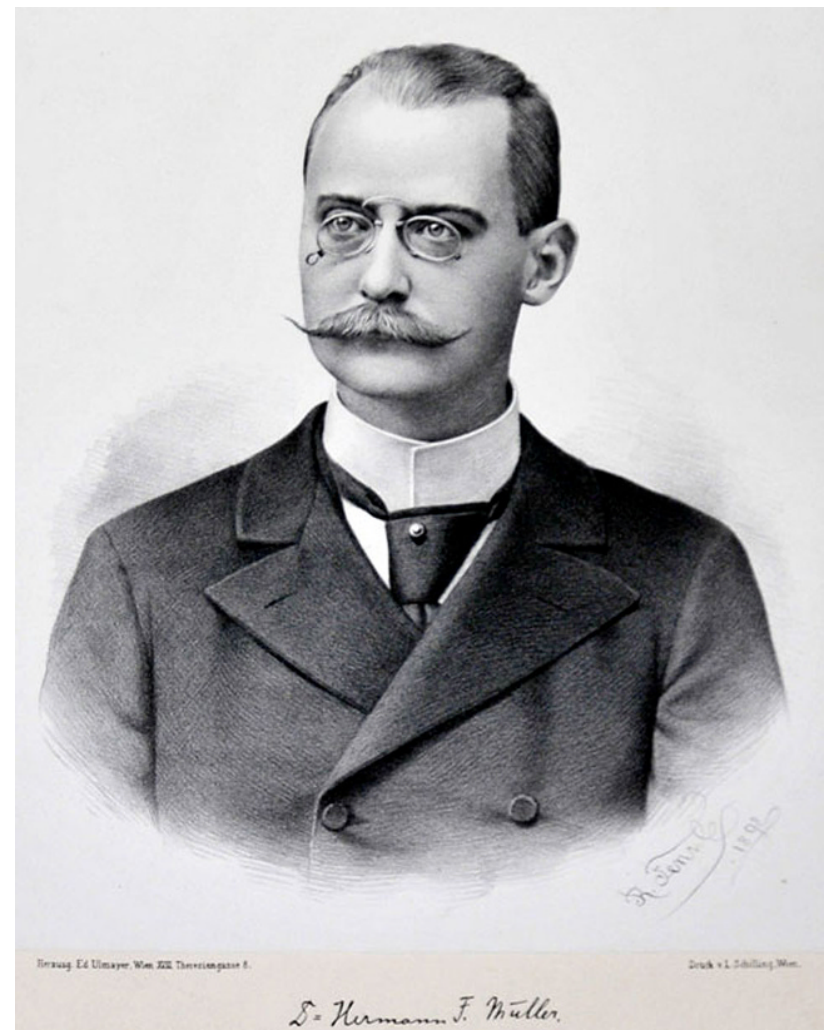

Abb. 1 Dozent Dr. Hermann Franz Müller, geb. am 25. Oktober 1866 in Oberdöbling (heute: Wien, 19. Bezirk). (MUWFO-IIR-003062-0001-001-01). Nach Promotion 1889 in Graz Aspirantentätigkeit in München und Wien, ab 1895 Assistent der I. Med. Klinik in Wien (Hermann Nothnagel), 1897 Habilitation für Interne Medizin. Am 23. Oktober 1898 Tod an der in Wien erworbenen Pest

Kenntnisse über den erst kürzlich (1894) während der oben genannten Epidemie in Hongkong gleichzeitig und unabhängig von einander vom Schweizer Alexandre Yersin (1863-1943) und Japaner Shibasaburo Kitasato (1852-1931) entdeckten Pesterreger. Österreich war damit der erste Staat, der eine Kommission zur Erforschung der Pest-Pandemie entsandte. Danach folgten die deutsche und die russische Regierung, deren Delegierte erst nach den Österreichern in Bombay eintrafen, nämlich Georg Gaffky, Richard Pfeiffer, Georg Sticker und Adolf Dieudonné am 8. März (und Robert Koch erst Mitte April) bzw. V. K. Wyssokowitz und Kirill Danilowitsch Zabolotny am 15. März [14, 15].

Nach umfänglichen Vorbereitungen verließ die österreichische Kommission am 3. Februar 1897, versehen mit reichlicher Ausrüstung an Bord des Eildampfers „Imperator“ der österreichischen LloydGesellschaft den Hafen von Triest. Von den zuständigen Ministerien war der österreichische Vertreter am Conseile sanitaire internationale gebeten worden, die Expedition von Port Saïd bis Suez zu begleiten, um sie bei etwaigen Untersuchungen in den zur Cholerabekämpfung bestehenden Quarantäne-Anstalten zu unterstützen.

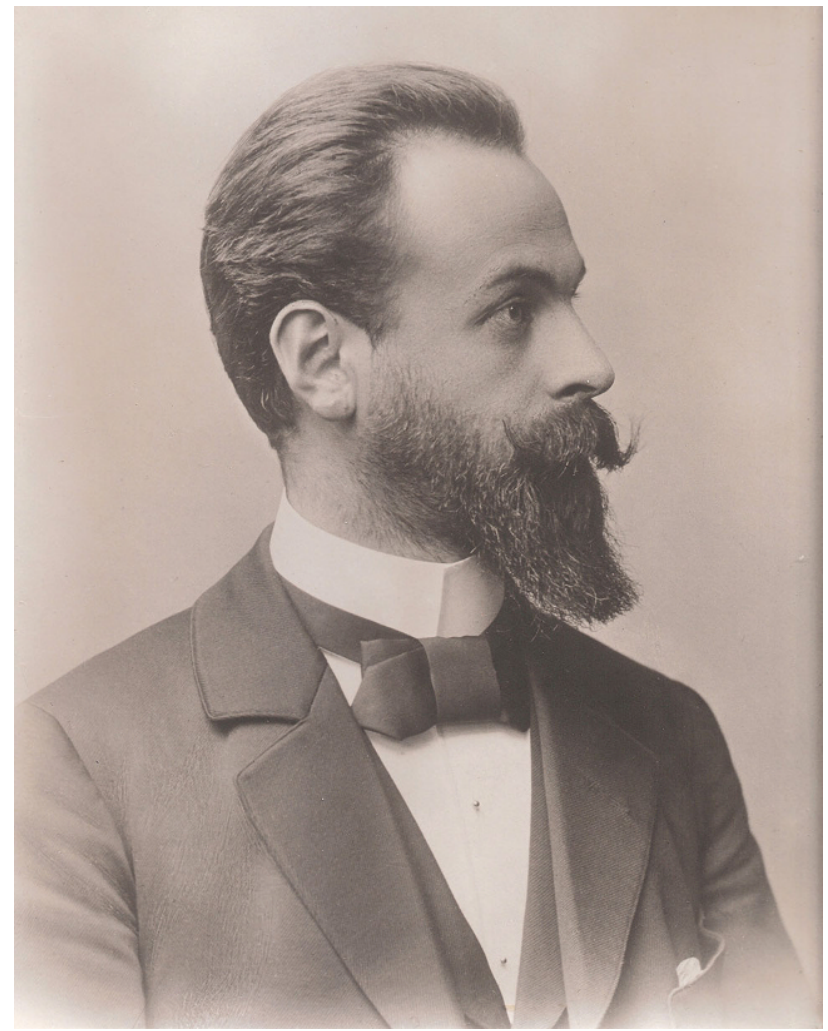

Abb. 2 Dr. Heinrich Albrecht, geb. am 24. Juli 1866 in Wien. (MUW-FO-IIR-004513-001). Nach Promotion 1891 in Wien Eintritt in das Anatomische Institut (Emil Zuckerkandl) und ab 1893 Assistent am Pathologisch-anatomischen Institut (Anton Weichselbaum). Nach Habilitation 1899 weiterhin bei Weichselbaum und zusätzlich Übernahme der Leitung der Prosektur mit Histologisch-bakteriologischem Institut der Wiener Poliklinik. 1902 a.o. Professor, 1903 Prosektor des Wiener Wilhelminen-Spitals, 1908 Prosektor des Kaiser-FranzJoseph-Spitals und gleichzeitig Vorstand des Universitätsinstituts für Pathologische Histologie sowie weiterhin in der Funktion in der Wiener Poliklinik. 1913 Vorstand des Instituts für Pathologische Anatomie in Graz und 1920 in Wien. Am 28. Juni 1922 Tod in Wien an der in Graz erworbenen Tuberkulose

Am 20. Februar 1897 erreichte die Kommission Bombay. „So hoffnungsvoll auch der Kommission das Feld ihrer Arbeit erschien, ebenso ungewiss waren aber die Aussichten auf Erlangung von Material, besonders da die Zeitungsnachrichten über den Widerstand der einheimischen Bevölkerung ärztlichen Massnahmen gegenüber berichteten. Dem Umstande jedoch, dass die Commission die erste am Platze war, verdankt sie eine glückliche Erreichung ihres Zieles.“ Dieses wurde auch durch das bereitwillige Entgegenkommen der englischen Behörden, insbesondere des obersten Gesundheitsbeamten Surg-Colonel Dr. Weir gefördert, wodurch der Kommission die Leichen des Arthur-Road-Hospitals zur Verfügung standen [17]. Arbeitsräume bekam die Kommission in einem monumentalen Schulbau im Stadtzentrum.

Das ziemlich isoliert liegende Arthur-Road-Hospital war für die Untersuchungen günstig eingerichtet. 


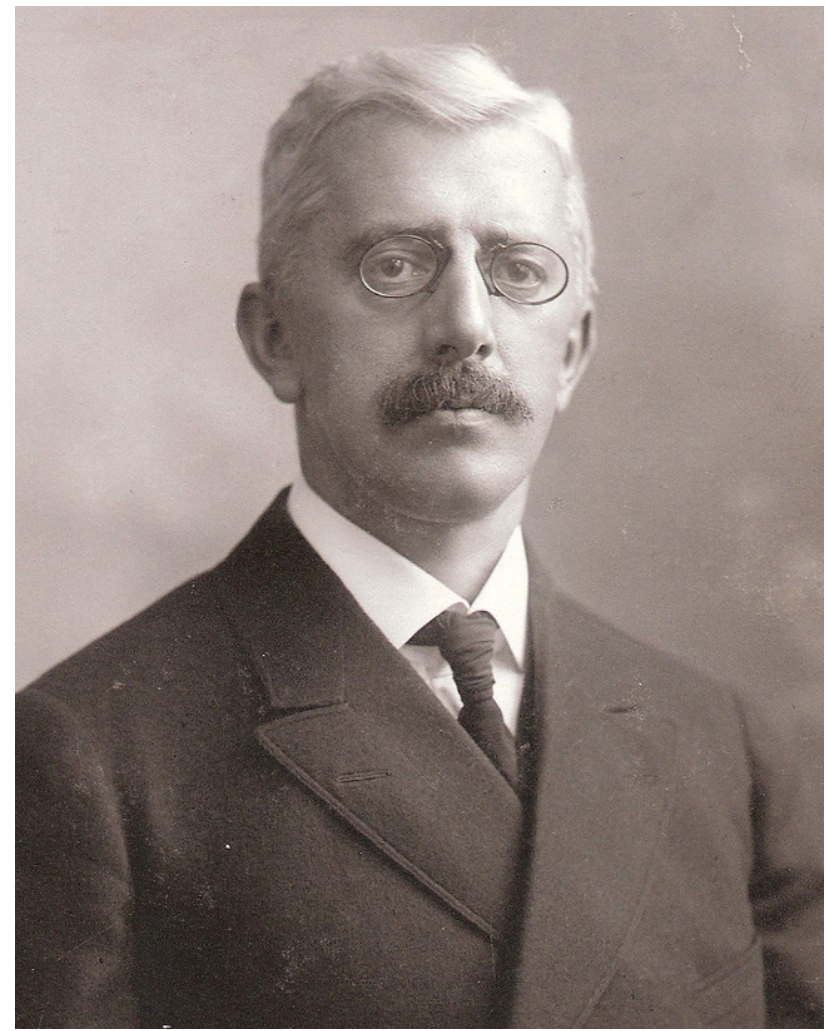

Abb. 3 Dr. Anton Ghon, geb. am 1. Jänner 1866 in Villach. (MUW-FO-IIR-001765-0001). Nach Promotion 1880 in Graz und Militärdienst arbeitete er als Volontär an der Dermatologischen Universitätsklinik in Wien (Isidor Neumann) und kurze Zeit als Allgemeinmediziner. 1892 trat er als Aspirant in die Prosektur der Krankenanstalt Rudolfstiftung ein und wurde 1892 Demonstrator am Institut für Pathologische Histologie und Bakteriologie (beide: Anton Weichselbaum). 1894 folgte er Weichselbaum als dessen Assistent an das Pathologischanatomische Institut der Universität Wien. Er habilitierte sich 1899 nach seiner Teilnahme an der Pestkommission. 1902 a. o. Professor. 1910 Vorstand des Pathologisch-anatomischen Instituts der Deutschen Universität in Prag. Ein Jahr nach der Emeritierung starb Ghon am 23. April 1936 in Prag an Tuberkulose und einem Herzwand-Aneurysma

Die dort versorgten Patienten litten an verschiedenen Stadien der Pest, manche genasen, andere starben während oder kurz nach der Einlieferung. Es konnten also bereits am 24. Februar die klinischen Tätigkeiten und fünf Tage danach die Obduktionen aufgenommen werden [14].

Der Delegierte Doz. Müller war ausschließlich für die klinischen Untersuchungen zuständig. Die Delegierten Dr. Albrecht und Dr. Ghon führten gemeinsam die pathologischen und bakteriologischen Arbeiten an den Patienten und den Verstorbenen durch. Dem Hilfsarzt Dr. Pöch wurden neben Hilfeleistungen für die beiden als sein selbständiger Arbeitsbereich die Blutuntersuchungen und das Fotografieren übertragen.

Patienten und der Pest Erlegene standen in den ersten Wochen reichlich für Untersuchungen zur Verfügung, doch nach dem Höhepunkt der Epidemie in
Bombay etwa Mitte März wurden sie ziemlich rasch immer spärlicher. Die insgesamt großen Erfahrungen wurden dadurch gewonnen, dass es „der Kommission gelungen war, sich sofort gleichsam in den Besitz des Arthur-Road-Hospitals zu setzen, welches sie auch gegen die später Angekommenen mit Erfolg vertheidigte, und dass sie gerade die erste Zeit ihres Aufenthaltes in Bombay, wo die Pest noch wirklich erschreckend wüthete, mit Anstrengung aller zu Gebote stehender Kräfte arbeitete“.

Die österreichische Delegation überließ der später eingetroffenen deutschen Delegation „einen erheblichen Theil des bis jetzt (Mitte April) gewonnenen Leichenmaterials“, was diese als freundliches Entgegenkommen würdigte [16].

Nachdem am 22. April mit der Abrüstung des Laboratoriums begonnen worden ist, begann die Rückfahrt am 1. Mai 1897 wieder mit dem Lloyd-Dampfer „Imperator“, jedoch diesmal unter der gelben Quarantäneflagge. Dies hieß, dass niemand unterwegs das Schiff verlassen durfte.

Zum Gesundheitszustand der Kommissionsmitglieder ist zu berichten, dass bei allen bald nach der Ankunft in Bombay die axillären Lymphknoten leicht anschwollen und schmerzten, was auch noch einige Zeit nach der Rückkehr anhielt. Dies wurde auf eine milde „Infection mit dem Pestgifte“ zurückgeführt. Ein Mitglied hatte vorübergehend Zeichen einer Vergiftung mit Sublimat, das bei der Arbeit zur Desinfektion verwendet worden ist.

\section{Die Arbeit der Kommissionsmitglieder nach der Rückkehr}

In der Früh des 17. Mai landete die Delegation in Triest und traf nach einer nächtlichen Reise am folgenden Morgen in Wien ein. Zwei Tage danach erstattete sie in der Sitzung der mathematisch-naturwissenschaftlichen Klasse der Akademie der Wissenschaften einen kurzen Bericht über die vorläufigen Ergebnisse. Es folgten die üblichen Vorstellungen bei verschiedenen hohen Persönlichkeiten.

Nach einem kurzen Urlaub war die nächste Aufgabe, eine von der Außenwelt möglichst abgeschlossene und für die Untersuchungen geeignete Arbeitsstätte zu finden. Dafür kam eigentlich nur das Prosekturgebäude des Wiener Allgemeinen Krankenhauses in Frage. Dort wurde ein zweifenstriges, mit Steinplatten gepflastertes Zimmer zur Verfügung gestellt, das mit keinem anderen Arbeitszimmer verbunden war. Dieses wurde unter größter Berücksichtigung der möglichen Gefahren eingerichtet, welche die dort Arbeitenden und die Umwelt durch die künftigen Experimente mit lebenden Pestbakterien treffen könnten. In diesem sogenannten „Pestzimmer“ mussten neben den täglichen Arbeiten auch die infizierten Versuchstiere gehalten werden. Es waren also auch in Wien die Arbeitsverhältnisse für die Laboratoriumsuntersuchungen sehr ungünstig und ein erfolgreicher Abschluss 


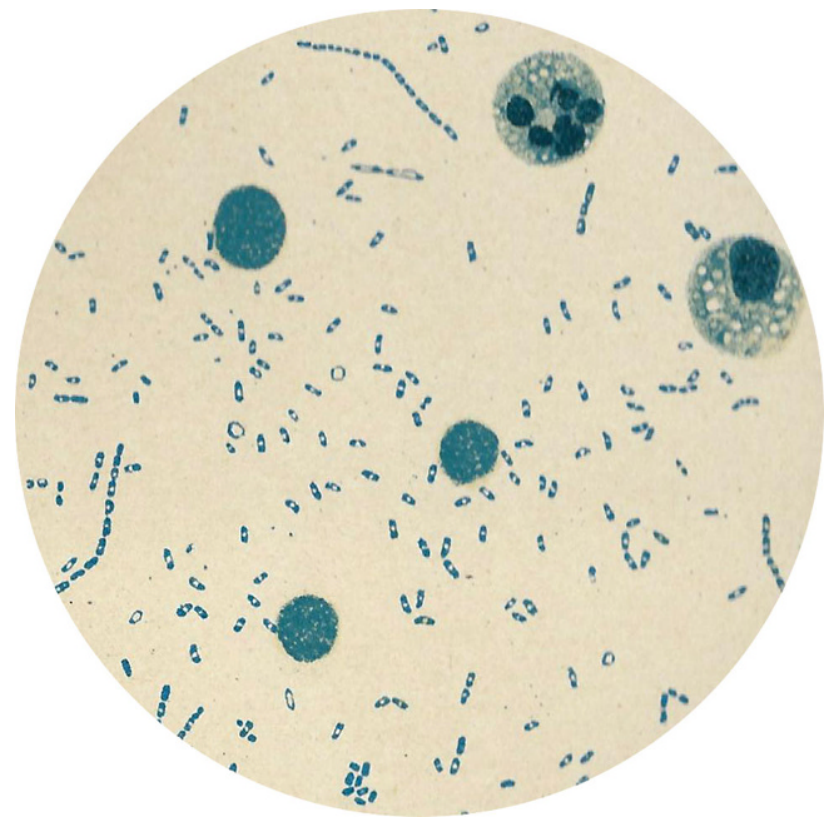

Abb. 4 Ausstreifpräparat vom Sputum bei primärer Pestpneumonie (Färbung mit alkalischem Methylenblau). Man sieht die typischen bipolar gefärbten Pestbakterien, zum Teil in Ketten angeordnet; daneben gibt es unregelmäßige Formen (am Bildrand rechts) [14: Tafel XIII/3]

wurde nur durch das stets bewiesene Interesse und die Unterstützung des Institutsvorstandes, Prof. Weichselbaum, ermöglicht.

Durch die Akademie der Wissenschaften wurden den Heimgekehrten folgende Aufgaben gestellt: 1. das in Bombay gesammelte pathologisch-anatomische Material wissenschaftlich $\mathrm{zu}$ bearbeiten, 2. durch Tierversuche festzustellen, wie und durch welche Eintrittspforten der Pesterreger in den Organismus eindringt und 3. ebenfalls durch Tierversuche festzustellen, ob und wie eine Immunisierung gegen Pest erzielt werden kann [17].

Da Albrecht und Ghon ihre Assistententätigkeit im Pathologisch-anatomischen Institut sowie Müller und Pöch an der I. bzw. II. Medizinischen Klinik wieder aufnehmen mussten, war die Zeit zur Bearbeitung der gestellten Fragen eingeschränkt. Zur Hilfe wurde ihnen der Prosekturdiener Franz Barisch zugeteilt, der als bisheriger Diener des bakteriologischen Laboratoriums des Pathologisch-anatomischen Instituts „die notwendigen Kenntnisse und Fertigkeiten besaß und mit den Gefahren seines Dienstes wohl vertraut war" und „sich im Dienste stets nüchtern erwiesen“ hatte. Er hat sich trotz der sehr drastischen Darstellung der Gefahren durch die Arbeiten mit den Pesterregern zu seinen neuen Dienstleistungen erklärt bereit und hielt dies auch nach den im Laufe der Arbeiten oftmaligen eindringlichen Wiederholungen aufrecht [19].

Die Berichte der Kommissionsmitglieder über die Auswertungen ihrer in Bombay erhobenen Befunde wurden in den Sitzungen der Mathematisch-naturwissenschaftlichen Klasse der Kaiserlichen Akademie der Wissenschaften am 3. Februar und 17. März 1898 vorgestellt, jene der in Wien angestellten bakteriologischen Kulturen und Tierversuche am 14. Dezember 1899; gemeinsam wurde alles im 66. Band der Denkschriften der Math.-naturwiss. Klasse abgedruckt.

Müller bespricht in seinem Bericht [20] die von ihm in Bombay bei 86 Pestkranken aufgezeichneten Krankengeschichten mit den Kurven von Fieber, Puls und Atemfrequenz. Dabei beschreibt er ganz ausführlich die klinischen Symptome aller Bereiche des Körpers, besonders die Bubonen der Lymphknoten sowie die Veränderungen der Lungen und der Haut. Epikritisch stellt er fest, „dass es sich bei der Pest - und zwar bei beiden bisher bekannten Formen, der Drüsen- und der Lungenpest - zuerst um eine örtliche und zunächst örtlich bleibende Infection handelt. Erst von der primären Entwicklungsstelle aus (Bubo, pneumonischer Herd, [Carbunkel]) kommt es zum Allgemeinleiden, zur Entwicklung der Pestmetastasen." Die bisher als das Häufigste angenommene primäre Infektion des Blutes, von welcher aus es erst zu lokalen Entwicklungen kommen soll, erscheint Müller Mangels bisher erbrachter Beweise als äußerst unwahrscheinlich. Unter der Annahme der zuerst örtlichen und zunächst so bleibenden Infektion muss - außer bei der primären Lungenpest durch Aufnahme der Erreger im Respirationstrakt - ein primärer Bubo entstehen, der in manchen Fällen weder klinisch noch anatomisch mit Sicherheit zu erkennen ist.

Die beiden Pathologen Albrecht und Ghon bearbeiteten die während der Obduktionen von ihnen diktierten und von Pöch geschriebenen Protokolle [17]. Weiters mussten die während oder sofort nach den Sektionen mit verschiedenen Methoden fixierten Organstücke im Wiener Institut histologisch untersucht werden. Der Ergebnisbericht wurde durch von Pöch angefertigte Fotografien einiger Kranker und mehrfarbige Abbildungen histologischer Präparate ergänzt (Abb. 4, 5 und 6).

Vom August 1897 bis Oktober 1898 führten Albrecht und Ghon ihre bakteriologischen Untersuchungen durch. Hierfür standen die während der Obduktionen von manchen Organen angefertigten Abklatschpräparate und bakteriologischen Kulturen zur Verfügung. Die Pestbakterien wurden hinsichtlich Morphologie, Anfärbbarkeit, kulturellen Verhaltens und Überlebensfähigkeit unter verschiedenen Bedingungen untersucht.

Wegen der damals, 1897/98, fehlenden Kenntnis, welche Tierarten empfänglich für den Pesterreger sind, wurden mehr als 750 Tierversuche angestellt. Hierfür wurden Meerschweinchen, Kaninchen, Ratten, Mäuse, Hunde, Hyänen, Katzen, Schweine, Affen, Vögel, Schlangen, Eidechsen und Frösche eingesetzt. In diesen Versuchen erwiesen sich nur Nagetiere, Meerschweinchen und Affen als empfänglich für Spontaninfektionen. Die Pathogenese glich der beim Menschen. Für die Übertragung der Pest interessant war der intensive Bakterienbefall der Darmzotten und 


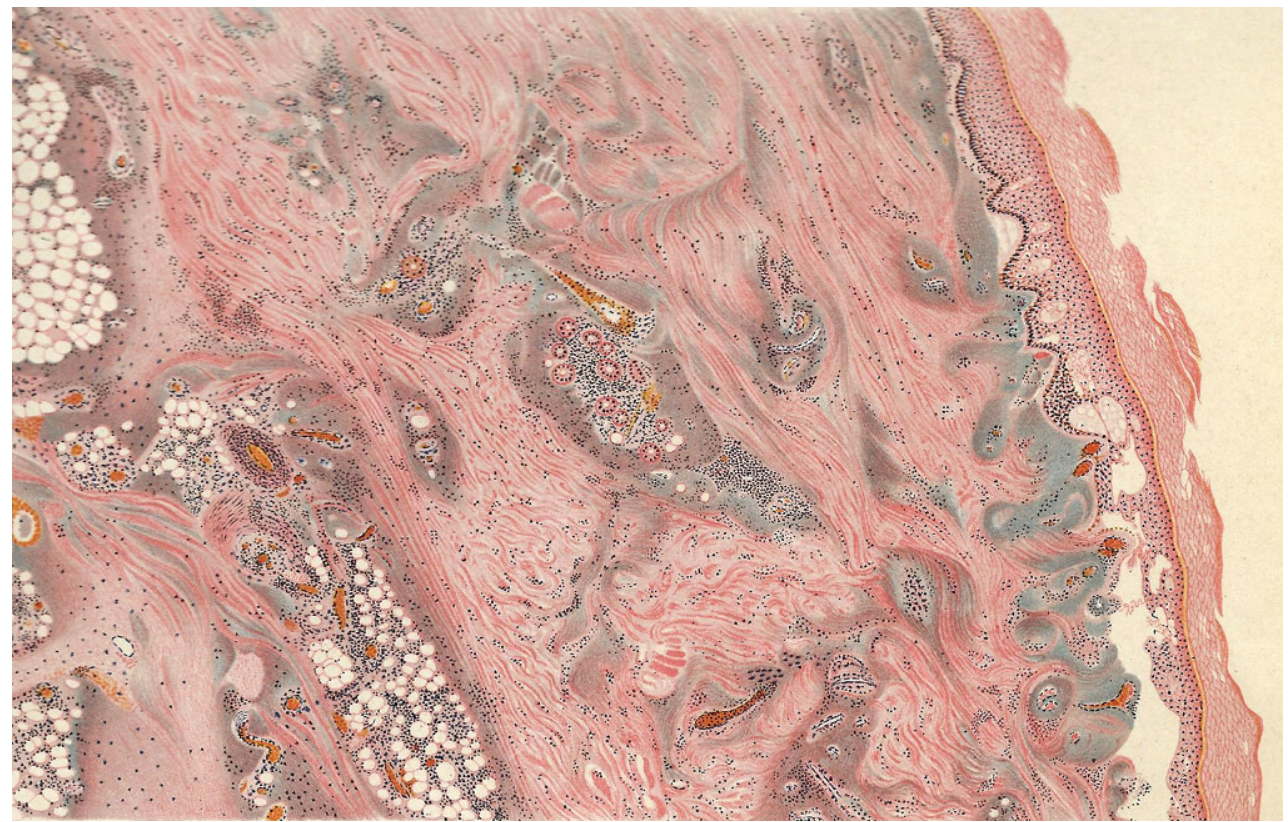

Abb. 5 Schnitt durch eine eingefallene Hautblase von der Innenfläche des rechten Unterschenkels samt den rötlich infiltriert aussehenden Schichten des Corium und subkutanen $\mathrm{Ge}-$ webes; fixiert am 7. Krankheitstag $4 \mathrm{~h}$ post mortem. Färbung mit Hämalaun-Eosin. Die blaue und bläulich- oder rötlichviolette Farbe zeigt enorme zusammenhängende Massen von

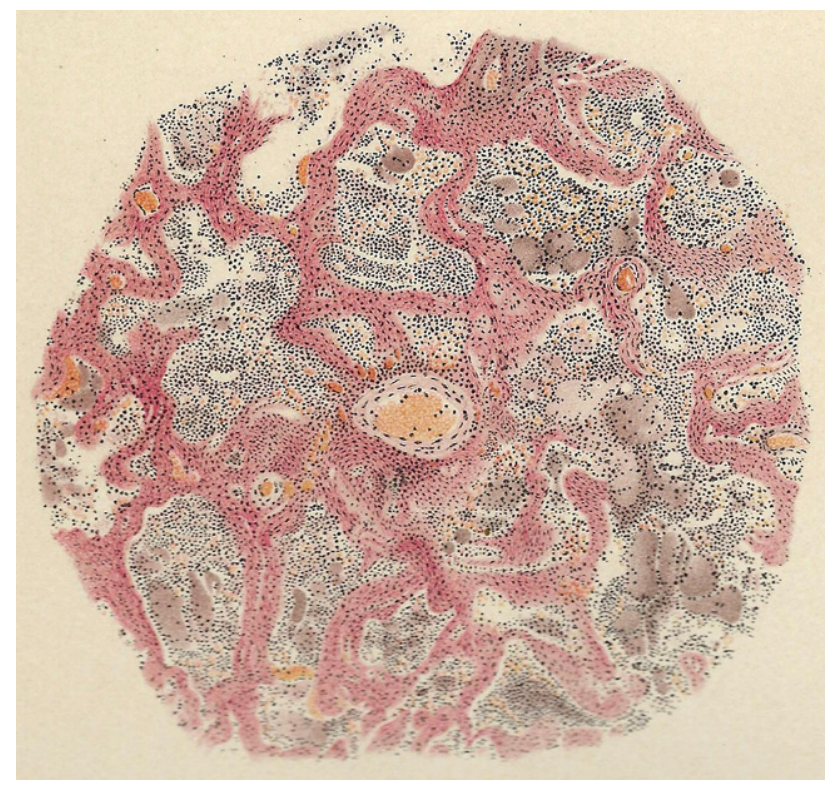

Abb. 6 Schnitt aus dem pneumonisch infiltrierten Oberlappen der rechten Lunge bei primärer Pestpneumonie; fixiert $3 \mathrm{~h}$ post mortem. Färbung mit Hämalaun-Eosin. Die Alveolarsepten sind in der für Pestpneumonie charakteristischen Weise in breite Stränge umgewandelt, die aus mit Eosin stark gefärbten Balken bestehen. Die bläulich-violetten Massen innerhalb der Alveolen sind große Anhäufungen von Pestbakterien [14: Tafel XII/1]
Pestbakterien. Der Inhalt der Hautblase ist zum größten Teil ausgeflossen. Die Coriumpapillen sind geradezu substituiert von einer Reinkultur von Pestbakterien. Desgleichen sind die Schweißdrüsen von einem breiten Mantel derselben umgeben. Auffallend ist das Fehlen von Hämorrhagien und größeren Leukozytenansammlungen [14: Tafel X/1]

nach deren Zerfall die massenhafte Ausscheidung mit dem Stuhl. Mehrfachinfektionen beim selben Tier waren möglich. Übertragungen durch Insekten wurden nicht beobachtet. Versuche zur Immunisierung von Ratten durch Injektionen steigender Mengen schwach virulenter Pestbakterien waren erfolgreich [18].

Die bakteriologischen Untersuchungen mussten Mitte Oktober 1898 wegen der folgenden Ereignisse abrupt abgebrochen werden.

\section{Die Pesttodesfälle in Wien 1898}

In der Zeit des planmäßigen Abschlusses der bakteriologischen Untersuchungen, als nur mehr ein am 4. Oktober mit Pestbakterien kutan infiziertes Meerschweinchen noch lebte, hatte sich der Diener Franz Barisch eine Pestinfektion zugezogen. „Im Allgemeinen war Barisch ernstlich bemüht, seine Dienstverrichtungen in der vorgeschriebenen Weise auszuführen“, er hatte „auch volles Verständnis für die Wichtigkeit der angeordneten Vorsichts- und Desinfektionsmaßregeln und sogar Interesse für die wissenschaftlichen Untersuchungen“. In der Nacht 14./15. Oktober erkrankte Barisch in seiner Wohnung im 4. Stock des sog. „Narrenturms“ im Allgemeinen Krankenhaus. Bei der Untersuchung durch den am Morgen des 15. Oktober herbei geeilten Ghon erklärte Frau Barisch, ihr Mann habe in der Nacht vom 8./9. Oktober „geschwärmt“ und sei am Morgen „erfroren“ nach Hause gekommen. Dies machte bei der Untersuchung durch Ghon gestellte Diagno- 
se Influenza verständlich. Bei der mikroskopischen Untersuchung des gewonnenen Sputums sah Ghon jedoch keine „Influenzabakterien“, sondern neben spärlichen „Pneumokokken“ eigentümliche Gebilde, die Degenerationsformen von Pneumonie- oder auch Pestbakterien sein könnten. Der zur Konsultation gerufene Müller hielt die Erkrankung von Barsch für eine beginnende krupöse Pneumonie. Da aber am nächsten Tag in den angelegten Sputumkulturen keine Pneumoniebakterien gewachsen waren und obwohl die mit 0,5 ccm Sputum intraperitoneal injizierte Ratte gesund geblieben war, steigerte sich für Ghon der Verdacht, dass die eigentümlichen Gebilde im Sputum doch Pesterreger sind und eine Pestinfektion bestehen könnte, was der zur Unterstützung gerufene Albrecht bestätigte. Doch auch bei einer abermaligen internistischen Untersuchung glaubte Müller nicht an eine Pestinfektion. Trotzdem bestand Ghon auf sofortiger Isolierung von Barisch. Müller organisierte dessen Transport mittels Tragbare in ein Isolierzimmer der I. Medizinischen Klinik. Dort wurden Barisch zur Pflege zwei „Wärterinnen“ zugeteilt: die erfahrene Johanna Hochecker und die Anfängerin Albine Pecha, die eigentlich gar nicht Krankenwärterin werden wollte. Die internistische Untersuchung lag weiterhin bei Müller. Am 17. Oktober wurde Müller das vom Prosektor der Krankenanstalt Rudolfstiftung, Prof. Dr. Richard Paltauf (1858-1924), übermittelte Pestserum [von Emile Roux (1853-1933), Pasteur-Institut] zur Injektion des Kranken übergeben und ihm empfohlen, auch sich und die Wärterinnen zu injizieren. Müller lehnte jedoch den Gebrauch des Serums ab.

Franz Barisch starb am 18. Oktober 1898 um 1/24 Uhr nachmittags noch immer mit der Diagnose einer krupösen Pneumonie. Erst am folgenden Morgen ergaben weitere vor dem Tode mit Sputum angelegte Kulturen und die Sektion der damit intraabdominal injizierten Ratte den Beweis der Pestinfektion [19, 21, 22]. Als Infektionsursache wurde eine Unachtsamkeit während der Tage vom 9. bis 12. Oktober angenommen, da Barisch erfahrungsgemäß einige Tage nach einem allerdings seltenen „Schwärmen“ [wie für die Nacht 8./9. Oktober berichtet] etwas ungeschickt und zerstreut zu sein pflegte.

Am 20. Oktober erkrankte die Wärterin Pecha. Sie wurde hochfiebernd von Müller gemeinsam mit der Wärterin Hochenecker in einem Infektionswagen in die „Exspektanzbaracke“ des Kaiser-Franz-Josef-Spitals geführt. Dort bezogen alle drei je ein Isolierzimmer. Für den Wartungsdienst wurden drei erfahrene Pflegeschwestern aus den etwa hundert im Hause tätigen Schwestern vom Heiligen Herzen Jesu ebenfalls in der Exspektanzbaracke untergebracht. Mit der Besorgung wurde der rangälteste Diener der Infektionsabteilung betraut. Entlang des die Baracke und den sie umgebenden Rasen gezogenen Kordons machten drei einander alle acht Stunden ablösende Wächter ihre Runden. Die isolierten Personen waren von jedem direkten Verkehr mit der Außenwelt abgeschlos- sen. Gespräche wurden über die Fenster geführt, angelieferte Gegenstände jeder Art und das Essen wurden nie Hand zu Hand übergeben. Es durften keine Gegenstände das Gebäude verlassen.

Nach der Ankunft am frühen Nachmittag dieses 20. Oktober erhob Müller den Status von Pecha und Hochecker und zeichnete die Befunde stenografisch selbst auf. Als am nächsten Morgen Müller über das Fenster durch einen Kollegen nach dem Zustand der Kranken gefragt wurde und diesen als schlecht bezeichnete, erschien er selbst bereits in reduziertem Zustand, der sich nach der von ihm gehaltenen Morgenvisite verschlechterte. Es stellten sich hohes Fieber und Husten mit reichlichem charakteristischem Auswurf ein. Die nun untersuchten Sputa von Pecha und Müller zeigten das für Pest typische Bild.

Am 22. Oktober wurde Pöch zur Behandlung zugezogen. Vor Antritt seiner Arbeit wurde er genau über die Isolierungsmaßnahmen unterrichtet und erhielt eine Injektion von $10 \mathrm{ccm}$ Pestserum in die Unterbauchgegend. Danach wurde ihm und der ihm zugeteilten geistlichen Schwester die nahe zur Exspektanzbaracke liegende „Blatternbaracke“ als Wohnung zugewiesen.

Pöchs Morgenuntersuchung der beiden Kranken am 22. Oktober zeigte hohes Fieber mit allgemeiner Beeinträchtigung. Müller verweigerte jede ärztliche Behandlung und alle Medikamente. Pecha war bewusstlos. Abends war der Zustand beider verschlechtert. Auch Hochecker hatte Fieber ohne sonstige Erscheinungen.

Doz. Dr. Hermann Franz Müller starb in der Früh des 23. Oktober 1898 um 1/25 Uhr. Seine Leiche wurde in einem verlöteten Metallsarg am nächsten Tag in aller Frühe um 5 Uhr abgeholt und zum Wiener Zentralfriedhof überführt, wo er sofort unter Ausschluss der Öffentlichkeit beigesetzt wurde. Sein Grab [heute: Gruppe 37-4-122] wurde später als Ehrengrab adaptiert.

Pöch kümmerte sich aufreibend um die beiden Isolierten. Hochecker hatte weiterhin Fieber. Pestbakterien waren nur bei Pecha nachweisbar. Ihr Zustand wechselte entsprechend dem Temperaturverlauf. Ab Mitternacht des 23. Oktober erhielt sie bis $\mathrm{zu}$ ihrem Tod insgesamt $580 \mathrm{ccm}$ eines Pestserums des PasteurInstituts, das auf Anordnung von Roux durch dessen Mitarbeiter Alexander Marmorek (1865-1923) nach Wien gebracht worden war. Nach einer deutlichen Verschlechterung des Zustandes erlag Albine Pecha am Morgen des 30. Oktober 1898 um 1/43 Uhr ihrer Infektion. Sie wurde neben dem Grab von Müller zur ewigen Ruhe bestattet. (Die Krankengeschichten der drei Gestorbenen findet man bei [19-23]).

Johanna Hochecker, deren Fieber Folge eines Lungenspitzenkatarrhs war, konnte frei von einer Pestinfektion aus der Isolierung entlassen werden. Auch Pöch ist nicht von der Pest erfasst worden. 


\section{Die Folgen der drei Wiener Todesfälle}

Die Gesellschaft der Ärzte in Wien hielt am 28. Oktober 1898 eine Trauersitzung. In dieser verlas der Präsident, Prof. Rudolf Chrobak (1843-1910), die von Müllers Lehrer, Prof. Hermann Nothnagel (1841-1905), verfasste Gedenkrede, der noch Ansprachen des Ministers Wilhelm Hartel und des Präsidenten der Akademie der Wissenschaften, Prof. Eduard Süß (1831-1914) folgten. Zum Gedenken an Müller als das Vorbild für kommende Generationen von Ärzten wurden Geldspenden für die Errichtung eines Denkmals gesammelt, das im 9. Hof des Wiener Allgemeinen Krankenhauses errichtet wurde. Darauf ehrt der Künstler Richard Kauffungen den Verstorbenen auf einem Relief unter seiner Büste durch die Darstellung der von Livius berichteten Selbstaufopferung des noblen Marcus Curtius für das allgemeine Wohl.

Auf dem Gebiet der Volksgesundheit wurden viele Maßnahmen zur Verhütung einer allfällig eingeschleppten Pest angeordnet, die zum Teil über das Ziel hinausschossen und eine Beunruhigung mancher Kreise verursachten. So wurden in den Sitzungen des Abgeordnetenhauses ab dem 20. Oktober 1898 nach der Beantwortung einer Interpellation in dieser Sache durch den Ministerpräsidenten in ausgedehnten Debatten temperamentvoll geredet und durch Beiträge mancher Abgeordneter die eigene Persönlichkeit dargestellt, wie z.B. durch das Verlangen eines allgemeinen Verbots bakteriologischer Untersuchungen [24]. Die Wiener Tageszeitungen haben sich natürlich der interessanten Ereignisse angenommen. So berichtete die „Neue Freie Presse“ sehr ausführlich und offenbar bemüht, die jeweils aktuelle Lage objektiv darzustellen. Das „Deutsche Volksblatt“ brachte täglich in den Morgen- und Abendausgaben in wesentlich umfangreicheren Beiträgen, seiner politischen Linie entsprechend, meist sehr einseitige und aggressive Darstellungen, welche die Ärzte und ganz besonders Nothnagel angriffen. Der Reichstagsabgeordnete und Wiener Bürgermeister Dr. Karl Lueger äußerte in der Debatte über das Züchten von Pestbakterien in Wien seine Meinung, dass das Allgemeine Krankenhaus, das aus der Zeit Kaiser Josef II. stammt, damals etwas ganz Außerordentliches war, der Jetztzeit nicht mehr entspricht und in modernem Stile anderswo aufgebaut wird.

Von all den positiven Folgen, die eine Flut von Anordnungen für die Statthaltereien und andere Dienststellen brachten, sei hier nur die vom OSR am 8. Juli 1899 beschlossene „Belehrung über die Pest und die sanitären Massnahmen zur Verhütung und Tilgung derselben“ erwähnt [25]. Diese wurde vom k.k. Ministerium des Inneren für alle politischen Landesstellen als verbindlich erklärt und ist in allen Landesstellen mit Ausnahme von Wien, Linz und Salzburg in die Landessprachen des jeweiligen Verwaltungsgebietes zu übersetzen [26].
Das von Müller nicht mehr vollendete Manuskript des umfänglichen Werkes „Die Pest“ [27] wurde von Pöch durch die von ihm verfassten Kapitel „Bakteriologie“, „Epidemiologie“, „Haut“ und „Schutzimpfung und Serumbehandlung" ergänzt.

Als Schlusswort der zahlreichen Debatten und Diskussionen hätte die Wortmeldung des von der Notwendigkeit und Nützlichkeit der bakteriologischen Forschungen überzeugten Mitgliedes des Abgeordnetenhauses, Wrabetz [22], dienen können: „wenn die Regierung mittels eines Nachtrages zum Budget Summen beanspruchen würde, die den besprochenen $\mathrm{Zu}$ ständen eine Remedur schaffen könnten, dann ist Dr. Müller nicht umsonst gestorben, dann kann man ihn als den Begründer der modernen bakteriologischen Institute und Krankenhäuser Wiens ansehen“. Hier sei auch der in ihrem 22. Lebensjahr gestorbenen Albine Pecha gedacht, die gar nicht Krankenpflegerin werden wollte und trotz ihrer Angst die freiwillig übernommene Verpflichtung bis zu ihrer eigenen Pesterkrankung erfüllt hat. Für ihr bleibendes Gedenken sorgten keine Trauersitzung und kein Denkmal.

Danksagung Mein Dank geht an das Josephinum, Ethik, Sammlungen und Geschichte der Medizin der Medizinischen Universität Wien für die Abb. 1, 2 und 3 und an Univ.Prof. Dr. Gerold Stanek für die Erklärung der Darstellung auf dem Denkmal für Doz. Müller.

Interessenkonflikt H. Flamm gibt an, dass kein Interessenkonflikt besteht.

Open Access Dieser Artikel wird unter der Creative Commons Namensnennung 4.0 International Lizenz (http:// creativecommons.org/licenses/by/4.0/deed.de) veröffentlicht, welche die Nutzung, Vervielfältigung, Bearbeitung, Verbreitung und Wiedergabe in jeglichem Medium und Format erlaubt, sofern Sie den/die ursprünglichen Autor(en) und die Quelle ordnungsgemäß nennen, einen Link zur Creative Commons Lizenz beifügen und angeben, ob Änderungen vorgenommen wurden.

\section{Literatur}

1. Hirsch A, Sommerbrodt M. Mitteilungen über die Pest-Epidemie im Winter 1878-1879 im russischen Gouvernement Astrachan. Berlin:Carl Heymann; 1880.

2. Raettig H. Die Pestpandemie des 20. Jahrhunderts. In: v. Rodenwaldt E, Jusatz HJ, Hrsg. Welt-Seuchen-Atlas. Bd. III. Hamburg: Falk-Verlag; 1961.S.31.

3. Raettig H, Felten H, Langer R. Pest in Europa 1899-1952. In: v. RodenwaldtE, Jusatz HJ, Hrsg. Welt-Seuchen-Atlas. Bd. II. Hamburg: Falk-Verlag; 1956. S. 25-7.

4. Kobler G. DieVenediger Pest-ConferenzimLichte der neueren Forschungen. Wien Med Wochenschr. 1898;58:894-9.

5. Anonym. Die Beulenpest im Jahre 1897-1898. Das österr Sanitätswes. 1898;10:218-20.

6. Anonym. Verhandlungen des k. k. Obersten Sanitätsrathes. Das österr Sanitätswes. 1896;8:421-2.

7. Anonym. Pest-Nachrichten. Das österr Sanitätswes. 1896;8:464.

8. Anonym. Der Pestfall in Triest. Das österr Sanitätswes. 1896;8:448-50. 
9. Anonym. Erlass der k. k. n.-ö. Statthalterei vom 22. Jänner 1897 betreffend die Assanierung in den Gemeinden anlässlich der Pestgefahr. Das österr Sanitätswes. 1897;9:35-6.

10. Anonym. Erlass des k. k. Ministeriums des Inneren vom 19. März 1807 an alle politischen Landesbehörden, betreffend Massnahmen gegen Einschleppung der Beulenpest. Das österr Sanitätswes. 1897;9:105-6.

11. Anonym. Mittheilungen über sanitäre Verhältnisse und Verfügungen im Auslande. Das österr Sanitätswes. 1897;9:113-4.

12. Anonym. Verhandlungen des k. k. Obersten Sanitätsrathes. Das österr Sanitätswes. 1897;9:25-6.

13. Gesellschaft der Ärzte. Geschichte der Gesellschaft der Ärzte in Wien 1837-1937. Wien: Springer; 1938. S. 112.

14. Albrecht H. Zur Geschichte der österreichischen Pestcommission. In: Über die Beulenpest in Bombay im Jahre 1897. (=Denkschriften d. Math.-naturwiss. Klasse d. Kais. Akademie d.Wiss., Bd.66). Im „Besonderen Abdruck“: Bd.I.Wien: Hof- und Staatsdruckerei; 1898. S. I-XIII.

15. Dieudonné A, Otto R. Pest. In: Kolle W, Kraus R, Uhlenhut P, Hrsg. Handbuch der pathogenen Mikroorganismen. Bd. 4. Jena, Berlin, Wien: Fischer und Urban \& Schwarzenberg; 1928. S. 182.

16. Mitteilungen der Deutschen Pestcommission aus Bombay vom 19. März d. J. Deut. Med. Wochenschr., 1897;33, Sonderbeilage zu No. 17.

17. Albrecht H, Ghon A, Pöch A. Pathologisch-anatomische Untersuchungen mit Einschluss der pathologischen Histologie und Bacteriologie. In: Über die Beulenpest in Bombay im Jahre 1897. (=Denkschriften d. Math.-naturwiss. Klasse d. Kais. Akademie d. Wiss., Bd. 66). Bd. IIB. Wien: K. k. Hofund Staatsdruckerei; 1898.
18. Albrecht H, Ghon A. Bacteriologische Untersuchungen über den Pestbacillus. In: Über die Beulenpest in Bombay im Jahre 1897. (=Denkschriften d. Math.-naturwiss. Klasse d. Kais. Akademie d. Wiss., Bd. 66). Bd. IIC. Wien: K. k. Hofund Staatsdruckerei; 1900.

19. Weichselbaum A. Bericht über die Infection des Dieners am pathologisch-anatomischen Institute Barisch mit Pestbacillen. Jahrbuch d. Wiener k. k. Krankenanstalten, Bd. 7. 1898. S.3-9.

20. Müller HF. Klinische Untersuchungen. In: Über die Beulenpest in Bombay im Jahre 1897. (=Denkschriften d. Math.naturwiss. Klasse d. Kais. Akademie d. Wiss., Bd. 66). Bd. IIA. Wien:K. k. Hof- und Staatsdruckerei; 1898.

21. Schilder S, Fischer I. Die Laboratorium-Pestfälle in Wien im Jahre 1898. Wiener medizingeschichtl. Beiträge, Bd. 2. Wien:Verl.d.Ars Medici; 1935.

22. Anonym. Die Pesterkrankungen in Wien. Wien Klin Wochenschr. 1898;11:981-4.

23. Müller HF, Pöch R. Die Pest. In: Nothnagel H, Hrsg. Specielle Pathologie und Therapie. Bd. V. Wien: Alfred Hödler; 1900. S. 131.

24. Anonym. Die Krankengeschichten von F. Barisch, H. F. Müller und A. Pecha. Jahrbuch d. Wiener k. k. Krankenanstalten, Bd.7.1898. S. 18-24.

25. Anonym. Die Pestfälle in Wien. Das österr Sanitätswes. 1898;10; Beilage zu Nr. 45:31-45.

26. Oberster Sanitätsrat. Belehrung über die Pest und die sanitären Massnahmen zur Verhütung und Tilgung derselben. Das österr Sanitätswes. 1899;11; Beilage zu Nr. 43: 143-54.

27. k. k. Ministeriums des Inneren. Erlass des k. k. Ministeriums des Inneren vom 12. November 1899, Zl. 38224. Das österr Sanitätswes. 1899;11:450. 